\title{
Plug Device
}

National Cancer Institute

\section{Source}

National Cancer Institute. Plug Device. NCI Thesaurus. Code C50108.

A device designed to seat into an opening in a device. 